\title{
Web and IoT-based hospital location determination with criteria weight analysis
}

\author{
Abeer Hadi, Mahmood Zaki Abdullah \\ Computer Engineering Department, College of Engineering, Mustansiriyah University, Baghdad, Iraq
}

\begin{tabular}{l}
\hline \hline Article Info \\
\hline Article history: \\
Received Oct 1, 2021 \\
Revised Dec 31, 2021 \\
Accepted Jan 14, 2022 \\
\hline
\end{tabular}

Keywords:

COVID-19

GPS

Internet of things

MCDM

TOPSIS

\begin{abstract}
The hospital location selection for COVID-19-infected patients is out to be one of the most critical decisions for healthcare sectors in high-case countries. In this study, optimal urban hospital location selection for COVID-19-infected patients has been done out of multiple alternative locations in city of Baghdad Iraq by introducing a web application system that can find the best site from alternatives by using MEREC and modified technique for order of preference by similarity to ideal solution (TOPSIS) algorithms. MEREC algorithm is utilized to obtain criteria weights and modified TOPSIS for ranking the alternatives. Four criteria are considered with eight alternatives sites. The proposed system has two-part, hardware part (embedded systems) designed by utilizing NEO-6M GPS receiver with ESP8266NodeMCU to obtain coordinate of regions and then, using the HTTP protocol to communicate to submit these data to database server. The second part is the web application developed by PHP, JavaScript, CSS, HTML, and MySQL used to allow the system admin to enter the locations of the alternatives with their criteria into the system to get the best urban hospital location for COVID-19-patients. The results showed effectiveness of overall suggested system and appropriateness of the modified TOPSIS method over the traditional TOPSIS method in ranking the alternative.
\end{abstract}

This is an open access article under the CC BY-SA license.

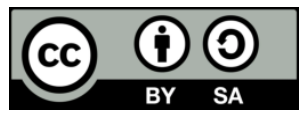

\section{Corresponding Author:}

Abeer Hadi

Computer Engineering Department, College of Engineering, Mustansiriyah University

Al-Bab Al-Muadham Street, Baghdad, Iraq

Email: egma019@uomustansiriyah.edu.iq

\section{INTRODUCTION}

The location selection process which requires analysis, identification, evaluation, and choice among alternatives, is affected by many qualitative and quantitative criteria or parameters. In this meaning, it can be clearly said that location selection represents a typical multi-criteria desition making (MCDM) problem [1], [2] and MCDM provides a collection of algorithms for determining the best choice [3]. As a result, it is easy to conclude that urban site selection is an MCDM challenge. MCDM methods have also been used for hospital location selection based on this knowledge. These studies essentially focus on determining multiple criteria for hospital location selection or any other urban scenario. The work in this research includes two main parts; the first part concentrates on IoT that is represented by geolocation of alternatives sites which are defined by using a design and implement geolocation system, the second part is represented by design and implement web application system to implement MCDM algorithms. It is obvious that there are no available researches aggregate the two parts with the same application. Moreover, utilizing the web application, so the literature survey will include similar researches regardless of the application type because the selected application in this work is merely a case study to verify the work on the prototype. 
Agrawal and Qadeer [4] presented geolocation system or tracking to tracke the path in real-time using phone, embedded system integrating Arduino Uno R3 microcontroller, Google Map on a mobile, global positioning system (GPS), and global system for mobile (GSM) communication technology. Internet of things (IoT) based intelligent transportation system is purposed in [5] which aims to navigate and locate position of the CIT college bus and to provide user understandable by Google Map API, MIT App Inventor, and Thingspeak cloud. The purposed systems are integrated with cloud technology and GPS and send the real-time live streaming data to a cloud server over hypertext transfer protocol (HTTP) protocol to send data. Luthfi [6] the researcher has presented an integrated tracking system hardware starting from neo-6 GPS module as a location determinant, a temperature sensor as a sensor value that will be sent to the IoT platform, and NodeMCU as a processor. This study uses the Thinkspeak platform to display GPS data with HTTP protocol as communication to send data. Mounika and Chepuru [7] suggested a system that is designed to track the vehicle when it is lost by GPS and GSM technique. GPS receiver and GSM module use Arduino UNO controller to forward the commands. GPS module will transfer the location values to the controller. The controller will receive it and sends that information to the automobile user using a GSM modem. Thingspeak is used to monitor the sensors data channel. a real-time tracking system proposed in [8] by designing a system for tracking and placing any vehicle. The design is embedded system utilizing by GPS and GSM that will constantly detect moving vehicle and provide its state on request.

Aytac et al. [9] the analytic hierarchy process (AHP) approach is suggested in this study to assist hotel management in selecting the most appropriate site for their new tourist hotel investment. In the application section, the computational process and efficiency of the AHP technique are demonstrated by selecting tourist hotel locations in Denizli Turkey. The researcher Kouchaksaraei et al. [10] tries to solve locating the problem of glasshouses by some important criteria that affect this issue. This research aimed at introducing new hybrid methods for glasshouse positioning based on step-wise-weight-assessment-ratioanalysis (SWARA), and complex proportional assessmen (COPRAS) method, SWARA is involved in evaluating criteria and COPRAS is utilized to evaluate alternatives, a case study in Tehran is presented in the research. Nazari et al. [11] technique for order preference by similarity to ideal solution (TOPSIS) technique was used in this study to find the best alternative for PV power plant installation in four distinct areas of Iran. The major locations for PV plant installation are four different places, and the optimum decision is determined using a MCDM technique called TOPSIS. The regions were chosen based on the need for power and the availability of land for this purpose. Some decision-making criteria were established, and seven experts were askeded to complete a questionnaire for criteria weight in order to get the optimal choice.presented a novel comprehensive approach in [12] for hotel locating based on new hybrid MCDM methods best worst method (BWM)- weighted aggragated sum product assesment (WASPAS) methods and a sustainability perspective a case study to estimating probable places of hotels tested for the Shahrekord city, Iran. To ensure that the chosen location is not only the best decision for today but also useful for the hotel sector in the future, it should be based on both present and future aspects. MCDM algorithms are especially suitable ways in making complex decisions in various areas. Based on the AHP method the study in [13] examined a decision support model for site selection to construct a new hospital, the research was based on six main criteria and 19 sub-criteria. All districts in the Mugla province were considered as alternatives Datça, Fethiye, Bodrum, Dalaman, Kavakldere, Koyce giz, Marmaris, Mente s e, Milas, Seydikemer, Ula, and Yata gan are among these districts. The possibilities were ordered based on a 1-9 Saaty scale. During the COVID-19 epidemic the study in [14] focused on determining the site of a temporary hospital,agray-based decision support framework was presented for the decision-making process considering the uncertain nature of the information related to location alternatives. Criteria importance through intercriteria correlation (CRITIC) and combined compromise solution (CoCoSo) methods were performed under gray values to determine the weights of criteria and to prioritize the location alternatives, selected five possible districts for the establishment of a temporary hospital in Istanbul. TOPSIS, evaluation based on distance from average solution (EDAS), and combinative distance-based assessment (CODAS) distance-based MCDM algorithms are used in [15] to solve the hospital site selection problem. The weights of criteria are determined using the CRITIC technique, and the overall ranking of hospital site options is determined using the methods CODAS, EDAS, TOPSIS, and methods.

As mentioned in previous studies of multi-criteria decision-making techniques, most of these studies depend on manual calculations. Also, the works of previous studies does not have a data storage system to update it continuously to achieve the desired goal, as it is designed for individual user interaction only one user has the ability to deal with proposed system. So MCDM algorithms were implemented using code programming with a graphical user interface (GUI) interface to reduce the time required for manual computation. In such systems, the user has the advantage of entering information for other alternatives and is flexible in dealing with and creating a reliable database in the matter of choosing the best urban site with the possibility of more than one user entering the system. All the steps executed time does not exceed 5 seconds, and it just needs to enter the parameters (criteria, coordinates, and alternative sites). So with this smart 
system, in a few minutes, we can find a more fitting new site, although it took more than a day in the normal way. Also, this study filled the gap in the literature by evaluating potential urban sites in the Iraq regions using new hybrid MCDM that combines modified TOPSIS with a newly developed MCDM method, called MEREC method programmed with PHP, JavaScript, CSS, HTML, and MySQL as a complete web application system based on IoT to locate the urban area.

\section{RESEARCH METHOD}

\subsection{Hardware system part (embedded systems)}

The structure of the suggested system design (embedded system) consists of ESP8266NodeMCU as a microcontroller, NEO-6M GPS module to get latitude, and longitude of the alternatives for the hospital location selection.

\subsubsection{ESP8266NodeMCU}

It is a microcontroller with built-in Wi-Fi capabilities [16], has $16 \times$ GPIOs (programmable general purpose input/output pins) that can be used to interact with all sensors and devices within the proposed system. It is small size, a cheap, and modern microcontroller unit which is built around an inexpensive system-on-a-chip (SoC) called the ESP8266 [16], [17]. Figure 1 shows the ESP8266 internal and pin out details. This ESP can be connected to the IoT platform by using Wi-Fi technology. The selected IoT platform of the system is the Thingspeak IOT platforms which can be used to monitor and control the sensor operate with HTTP protocol.

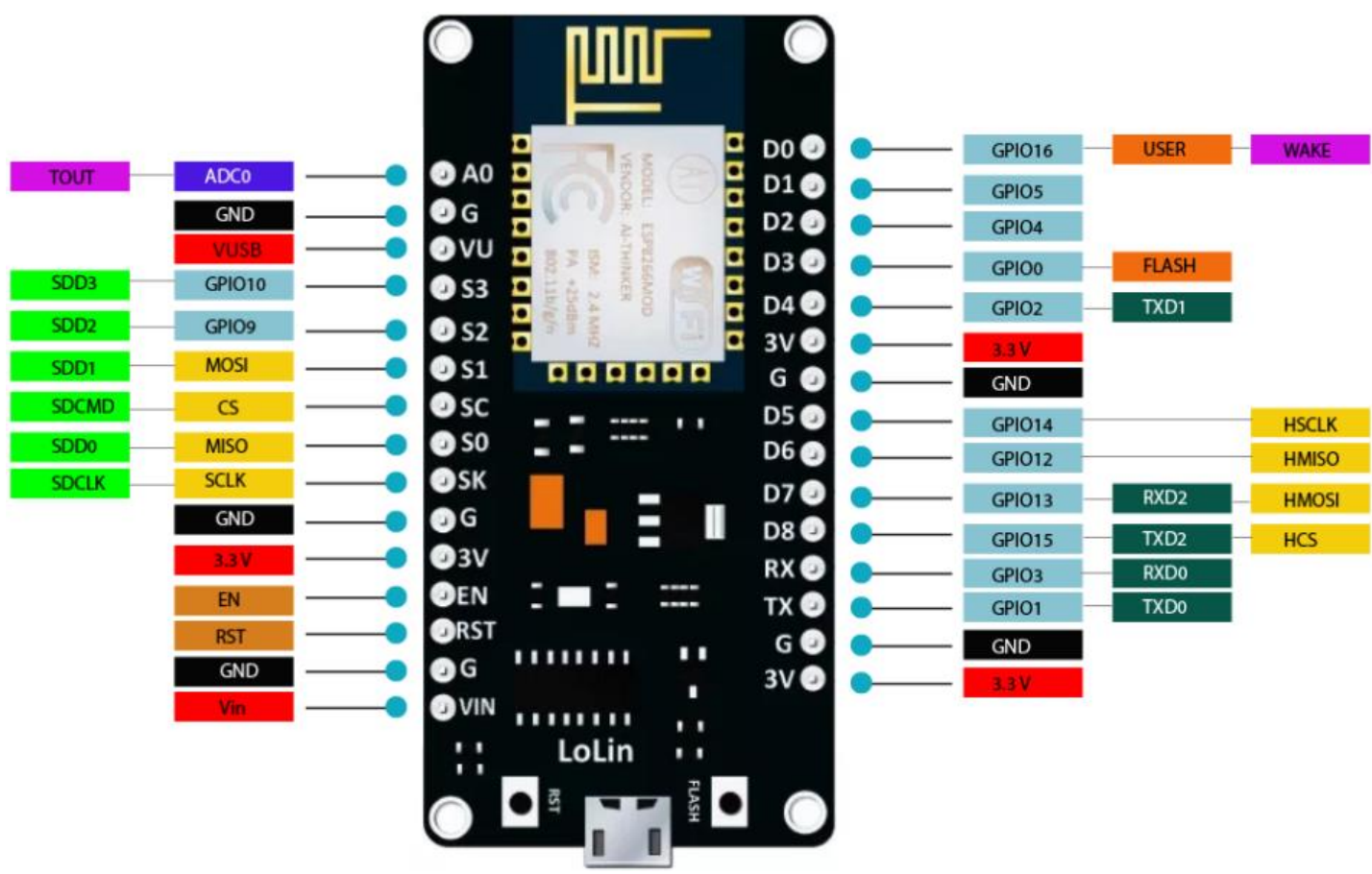

Figure 1. ESP8266 NodeMCU V3 pin out details

\subsubsection{NEO-6MGPS module}

The NEO-6M is a GPS module receiver that communicates with "GPS" satellites to determine its own location [5]. It then outputs its position's latitude and longitude as serial data. NEO-6M GPS receiver requires a power supply voltage $3 \mathrm{~V}$. It uses the NMEA protocol to transmit GPS data. The GPS module was chosen for its low power consumption, excellent localization accuracy, and built-in real-time clock for storing date and time information. The module includes a $5 \mathrm{~Hz}$ position update rate and an EEPROM with a backup battery to preserve configuration settings [6]. NEO-6MGPS module is seen in Figure 2. 


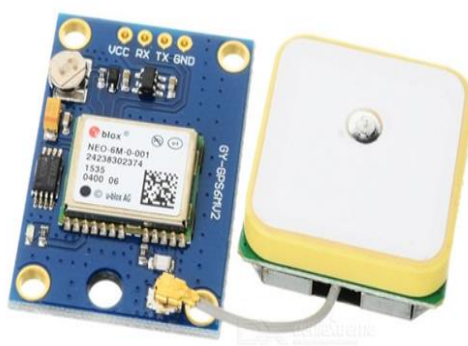

Figure 2. Neo-6m GPS module

\subsection{System architecture}

Figure 3 shows, the main elements of the suggested system is Neo-6m GPS which is utilized to get geographic coordinates [18] for any region. It is connected to Node MCU [19] by serial connection. So that Neo-6m sends the GPS [20] coordinates to Node MCU which is utilized to send GPS coordinates and update the location to a Thing-speak [21], [22] cloud (IoT platform) database using HTTP [23] as a communication protocol in real-time. Thing speak cloud continuously receives GPS coordinates every sec and plotted as graph, stores them in a database. Users can enter the web page platform (accessible via the Internet) to see current and historic data and manage the deployed solution. This architecture considers low-cost components, it is simple but functional for the specification of the project and makes the solution easy to deploy, easy to replicate, and scalable. In order to enable communication between the devices and the internet, HTTP protocol was supported in the geolocation device. The use of HTTP protocol is ideal for IoT applications, especially for communication with remote locations [24] where the network bandwidth is limited or when the node has memory constraints, it is ideal for simple communication between a node and a server.

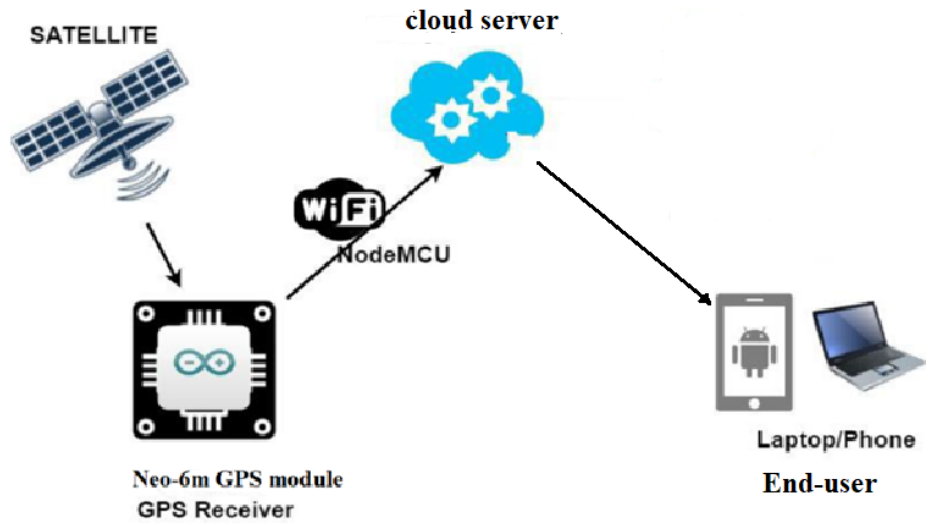

Figure 3. System architecture geolocation system

\subsection{Sofware part}

This part will explain the combined methodology applied in the study. The methodology consists of description hyprid MCDM algorithms, design and programming web application system.

\subsubsection{Merec method}

The "method based on the removal effects of criteria" (MEREC) method to calculate objective weights criteria [25], MEREC is suggested in this paper to measure the weights of the criterion in MCDM problem.

Depending on how each criterion affects the performance of alternatives when it is removed; more weight is given to the factors that have a larger influence on performance. The following are the MEREC method steps employed in this paper:

Step 1: create a decision matrix. A decision matrix is constructed at this stage, which shows the (m) scores or values for each criterion for each alternative (n). The letters $X_{i j}$ represent the constituents of this matrix. 


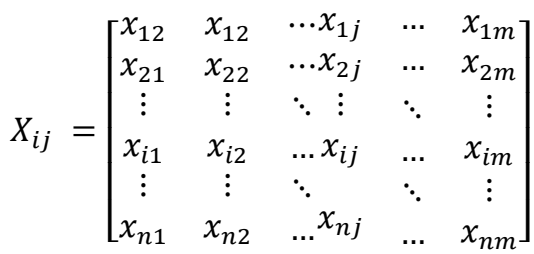

Step 2: Normalize the decision matrix $\left(X_{i j}\right)$. In this step, a simple linear normalization is used to scale the elements of the decision-matrix. The elements of the normalized matrix are indicated by $N_{i j}$. If B shows the set of "beneficial" criteria (the value must be max), and H represents the set of "non-beneficial" criteria (the value must be $\min ),(1)$ and (2) used for normalizing:

$$
\begin{aligned}
& N_{i j}=\frac{X_{j}^{\text {Max }}}{X_{i j}} \text { If } j \in B \\
& N_{i j}=\frac{X_{i j}}{X_{j}^{\text {Min }} \text { If } j \in H}
\end{aligned}
$$

Step 3: compute performance of the alternatives $\left(P_{\mathrm{i}}\right)$.

$$
P_{i}=\operatorname{In}\left(1+\left(\frac{1}{m} \sum_{j}\left|\operatorname{In}\left(N_{i j}\right)\right|\right)\right)
$$

Step 4. Remove each criterion from the equation to calculate performance of the alternatives.

$$
P_{i j}^{\prime}=\operatorname{In}\left(1+\left(\frac{1}{m} \sum_{k, k \neq j}\left|\operatorname{In}\left(N_{i k}\right)\right|\right)\right)
$$

Step 5: compute the total of absolute deviations.

$$
R_{j}=\sum_{i}\left|P_{i j}^{\prime}-P_{i}\right|
$$

Step 6: The final weights of the criteria is determine

$$
\begin{gathered}
w_{j}=\frac{R_{j}}{\sum_{k} R_{j}} \\
\sum_{j=1}^{n} w_{j}=1
\end{gathered}
$$

\subsubsection{Modified TOPSIS method}

The TOPSIS algorithm was interduced by Hwang and Yoon in 1981. Some of the benefits TOPSIS algorithm are simplicity, comprehensibility, rationality, good computational efficiency, as well as the capacity to quantify the relative performance of each choice in easy mathematical way. According to TOPSIS approach the optimum alternative is one that is close to a positive ideal solution (PIS) while being distant from a negative ideal solution (NIS) [26]. In this paper modified the traditional TOPSIS method by using the manhattan distance instead of the Euclidean distance in extracting the best alternative that is close to PIS due to the consistency of this knowledge in scientific application [27], [28] since use manhattan distance always gives the closest distance between two points. Euclidean distance always does not give shortest distance between points. The manhattan distance method has better performance than the euclidean distance [29]. So, used Euclidean distance to extract the worst alternative that is close to NIS it is done as in the traditional way. following are the modified TOPSIS method steps employed in this paper :

Step 1: calculate the normalized decision-making matrix $\mathrm{NN}=\left(\mathrm{NN}_{\mathrm{ij}}\right)_{\mathrm{m} * \mathrm{n}}$ where $N N_{i j}$ are normalized attribute values expressed as (8).

$$
N N_{i j}=\frac{X_{i j}}{\sqrt{\sum_{j=1}^{m} X_{i j}^{2}}} i=1,2, \ldots, n ; j=1,2, \ldots, m .
$$

Step 2: creat weighted normalized decision making matrix $\left[w n_{i j}\right]_{\mathrm{m} * \mathrm{n}}$. The weighted normalized value $w n_{i j}$ is calculate by (9): 


$$
w n_{i j}=w_{j} * N N_{i j}, i=1,2, \ldots, n ; j=1,2, \ldots, m
$$

Step 3: compute "PIS" $w n_{j}^{+}$"NIS" $w n_{j}^{-}$, respectively. If $C_{j}$ is a benefit attribute, then

$$
\left\{\begin{array}{l}
w n_{j}^{+}=\max \left(w n_{i j}\right) \\
w n_{j}^{-}=\min \left(w n_{i j}\right)
\end{array} i=1,2, \ldots \ldots, n ; j=1,2,3, \ldots \ldots m\right.
$$

If $C_{j}$ is a cost attribute, then

$$
\left\{\begin{array}{c}
w n_{j}^{-}=\min \left(w n_{i j}\right) \\
w n_{j}^{+}=\max \left(w n_{i j}\right)
\end{array} i=1,2, \ldots ., n, j=1,2,3, \ldots \ldots m\right.
$$

Step 4: compute distances of each alternative with $w n_{j}^{+}$and $w n_{j}^{-}$,

$$
\begin{aligned}
& f_{i}^{+}=\sum_{j=1}^{n}\left(w n_{i j}-w n_{j}^{+}\right) \text {manhattan distance } \\
& f_{i}^{-}=\left\{\sqrt[2]{\sum_{j=1}^{n}\left(w n_{i j}-w n_{j}^{-}\right)^{2}}\right\} \text { Euclidean distances }
\end{aligned}
$$

Step 5: Next, closeness coefficient $\left(c c_{i}\right)$ for each solution is computed by using the (14) and (15). Based on $\left(c c_{i}\right)$ values the alternatives are rank to select best alternative in descending order. the larger the $\mathrm{Q}$, the better the alternative.

$$
\begin{aligned}
& c c_{i}=\frac{f_{i}^{-}}{f_{i}^{-}+f_{i}^{+}} \\
& Q=\operatorname{Sort}\left(\left(c c_{i}\right)\right.
\end{aligned}
$$

\subsubsection{Haversine method}

The Haversine theorem is used to compute distances between two sites on the earth's surface based on latitude and longitude [30]. To compute the two distances, four variables must be prepared. The haversine formula, which provides a wide circular separation between two places on the surface of the sphere depending on longitude and latitude, is an essential navigation equation [31]. It is a way of calculating distance between two places that take into account the fact that the earth is not a plane but a curved plane with a radius of $6,367.45 \mathrm{~km}$.

\subsubsection{Web design}

Various web application development languages were employed to meet a variety of needs for the planned apps, resulting in a fully integrated system that allows users to benefit from it [32], [33]. The following is a summary of the overall goal of the proposed application: i) define and manage all information about number and values of criteria and alternatives by the system administrator; ii) create database for point 1 in MySQL database; iii) display the weight of the criteria; iv) display rank the alternatives; v) compute the distance between any two points on the map.

Web pages were designed using JavaScript, PHP, CSS and HTML [34] which perform functions and adds effects to the behavior of JavaScript, HTML pages and CSS implement the background functions such as login check, information validation as shown in Figures 4 and 5, and browsing function. Moreover, JavaScript embeds Google Map [35] on the website utilizing key provided by Google Company where region locations coordinat are displayed. The administration jobs include adding, deleting, browsing, and formatting into tables. Figure 6 represent home page for web-based system. 


\section{Register}

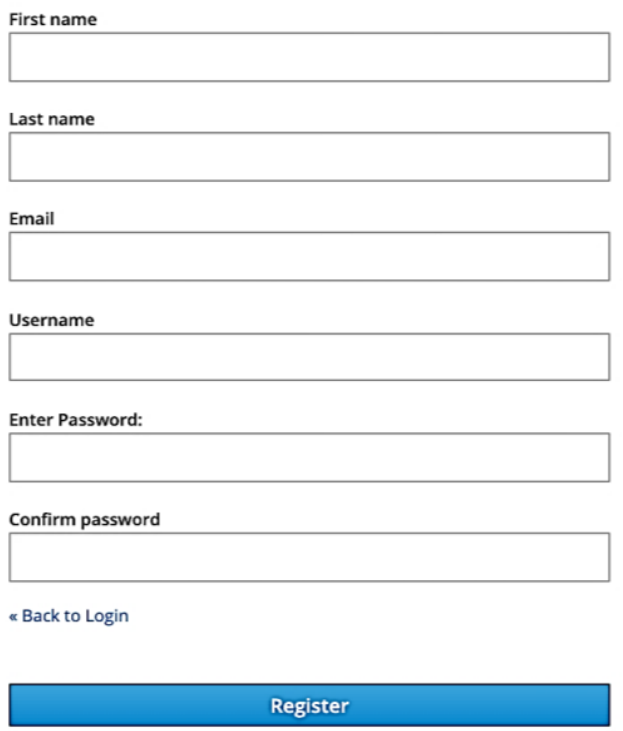

Figure 4. Register to system

\section{C $\oplus$ locahostllogin.php}

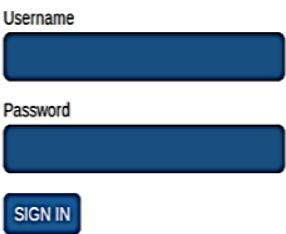

Figure 5. Login system

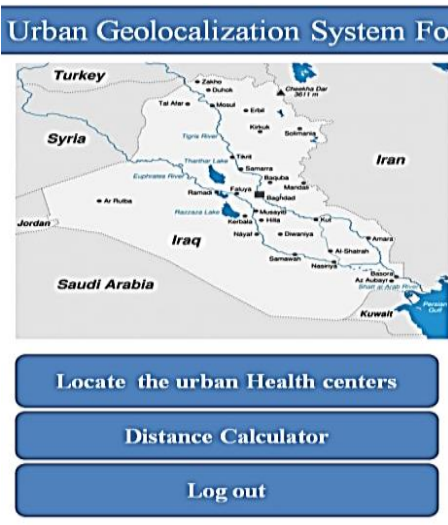

Figure 6. Home page for the web-based proposed system

\section{CASE STUDY}

This study was planned and conducted to solve the decision-making problem regarding selecting the best alternative (site) to build a new hospital for COVID-19 [36], [37] patients. The selection of the most suitable hospital place problem can be solved in following steps explained; Step 1 identification the research problem and defining the goal. Step 2 determine the set of alternatives. Step 3 determination of criteria by following literature reviews. Step 4 application of MEREC methodology to evaluate the importance of factors' weight. Step 5 the TOPSIS algorithm is utilized for ranking the optimal location for urban site selection. Eight sites were selected as a case study in Baghdad Iraq with four criteria [14] or components of the urban site have been challenged as shown in: i) distance to industrial sites (C1): measure distance between the hospital and industrial regions in relation to the fact that the emissions of factories in an industrial area can have a negative effect on the air quality level. The farther away from this event the better; ii) accessibility via airports (C2): measures the distance of the hospital to its closest international or local airport, which can be used to deliver patients in a critical situation; iii) distance from residential areas (C3): The distance from residential areas measures how far the hospital from areas with a high population density. The farther away from this event the better; iv) transportation/accessibility (C4): transportation (accessibility to hospital) accessibility via roads indicates how many different roadways are available in the district in order 
to deliver patients, the closer away distance to main streets' transportation way the better. The evaluation of each alternative based on the mentioned criteria is carried out using the harvison method.

\section{RESULTS AND DISCUSSION}

The system administrator (urban planner) login by entering a username and password and then creates the database of information related to all the alternative sites with their own criteria. eight alternatives sites have been chosen to determine the best of them as a case study in the Baghdad Iraq regions as in Table 1. Four criteria are some important parameters in hospital site establishment and defined distance in meters from these infrastructures. The weight of the criteria is calculated using the MEREC method then the TOPSIS algorithm is utilized to compute the ranks of the alternatives the. The results for the criteria weights after applying the MEREC method are represented in Table 2 the results show the correctness of the method in determining the weight and the consistency between the weights of the criteria. The results of applying the modified TOPSIS method for the alternatives are displayed in Table 3. According to the results of this study, the major priority for deciding the site of urban hospital for COVID-19 patients is alternative R7, the second priority is alternative $\mathrm{R} 6$, and the third priority is alternative $\mathrm{R} 8$.

The scenario proves the "efficiency" of the proposed system over the workflow. The results prove the performance of the proposed system in terms of management by allowing multiple users with authorization to use the web application. This application finds the best urban location based on Gps receiver and by using a web-based application that implements proposed MCDM algorithms. The web is designed to be interactive, easy to use, reliable, and secure in terms of authority. Moreover, a database is designed in a normalized pattern to avoid the redundancy in the data which exhausts the server. Both of the two platforms are scalable since it can be scaled enough to accommodate growing needs of data to initiate the implementation of the project idea in smart cities. The results also showed effectiveness of the proposed method modified TOPSIS to find the best urban areas comparative with traditional TOPSIS depending on the actual rank for sites. Modified TOPSIS showed match with the actual ranking of the sites unlike the traditional method, which showed an error in the arrangement of the alternative R8.

Table 1. Coordinates and Score the alternatives sites for each criteria

\begin{tabular}{ccccccc}
\hline Alternative & Latitude & Longitude & C1 $(\mathrm{m})$ & $\mathrm{C} 2(\mathrm{~m})$ & $\mathrm{C} 3(\mathrm{~m})$ & $\mathrm{C} 4(\mathrm{~m})$ \\
\hline R1 & 33.32081522 & 44.356666621 & 593.4 & 8831.09 & 88.34 & 248.65 \\
R2 & 33.31025723 & 44.34854469 & 526.6 & 8100.34 & 293.15 & 115.6 \\
R3 & 33.26907931 & 44.30075496 & 680.64 & 2552.01 & 119.74 & 300.72 \\
R4 & 33.31422694 & 44.33476167 & 672.41 & 6730.47 & 150.82 & 134.25 \\
R5 & 33.29372829 & 44.35512976 & 165.27 & 7542.13 & 83.82 & 364.79 \\
R6 & 33.28029072 & 44.22895472 & 7365.89 & 4371.31 & 1635.46 & 999.42 \\
R7 & 33.21956222 & 44.23661117 & 9978.3 & 5497.3 & 1012.67 & 726.42 \\
R8 & 33.16798327 & 44.34338471 & 597.69 & 13837.92 & 1425.98 & 914.49 \\
\hline
\end{tabular}

Table 2. Wieght criteria result from MEREC method

\begin{tabular}{cccccc}
\hline criteria & $\mathrm{C} 1$ & $\mathrm{C} 2$ & $\mathrm{C} 3$ & $\mathrm{C} 4$ & Sum \\
\hline $\mathrm{W}=$ & 0.352307224011 & 0.153548338003 & 0.280923188106 & 0.21322124988 & 1 \\
\hline
\end{tabular}

Table 3. TOPSIS method results

\begin{tabular}{ccccc}
\hline Alternative & CC $_{\mathrm{i}}$ & Traditional TOPSIS rank & Modified TOPSIS rank & Actual rank \\
\hline R1 & 0.243773030773 & 7 & 7 & 7 \\
R2 & 0.291576286666 & 3 & 4 & 4 \\
R3 & 0.276583094976 & 6 & 6 & 6 \\
R4 & 0.281793250031 & 5 & 5 & 5 \\
R5 & 0.218587191762 & 8 & 8 & 8 \\
R6 & 0.672937588556 & 2 & 2 & 2 \\
R7 & 0.742314132572 & 1 & 1 & 1 \\
R8 & 0.339996606155 & 4 & 3 & 3 \\
\hline
\end{tabular}

\section{CONCLUSION}

The urban hospital location selection problem is one of most significant choices in the healthcare sector in large cities. A bad location selection procedure may cause a slew of problems for the health workers and patients, as well as needless expenses for healthcare organizations. During the previous months, the COVID-19 epidemic had a notable impact on people's life and healthcare service quality. This paper 
proposed a web application system with a hybrid MCDM algorithms based on the MEREC and modified TOPSIS techniques for selecting the optimal urban hospital location for COVID-19-infected patients based on the internet of things. The system proposed uses HTTP protocol as a communication tool for data sending from embedded system to IoT platform. The proposed system was designed to allow the system administrator with a type of authorization to control the web application to enter the information about alternatives and criteria to run the proposed algorithms MEREC and modified TOPPSIS after that displays locations of regions automatically on Google map. A real-world case study from regions Baghdad Iraq was introduced to illustrate the hybrid MCDM approach embedded in the web application. The result of applying the proposed system showed that the 'R7' region from eight alternatives in Baghdad is the superior alternative for urban hospital site selection for COVID-19-patients. The results prove effectiveness of the proposed hybrid method (MEREC- modified TOPSIS) in finding urban sites.

\section{REFERENCES}

[1] B. Z. Yuksek and U. Dakeev, "Management of Urban Parking Lot Energy Efficiency with the Application of Wind Turbine and LED lights," Bulletin of Electrical Engineering and Informatics, vol. 3, no. 1, pp. 9-14, 2014, doi: 10.12928/eei.v3i1.180.

[2] C. Meshram and S. S. Agrawal, "Fuzzy Multi-criteria Decision Making associated with Risk and Confidence Attributes," Bulletin of Electrical Engineering and Informatics, vol. 4, no. 3, pp. 231-240, 2015, doi: 10.11591/eei.v4i3.506.

[3] I. Prasetyaningrum, K. Fathoni, and T. T. J. Priyantoro, "Application of recommendation system with AHP method and sentiment analysis," TELKOMNIKA (Telecommunication, Computing, Electronics and Control), vol. 18, no. 3, pp. 1343-1353, 2020, doi: 10.12928/TELKOMNIKA.v18i3.14778

[4] T. Agrawal and M. A. Qadeer, "Tracing Path with Arduino Uno using GPS and GPRS/GSM," 2018 International Conference on Computing, Power and Communication Technologies (GUCON), 2018, pp. 1203-1208, doi: 10.1109/GUCON.2018.8674953.

[5] V. Arun and M. Poongothai, "Implementation of IoT Based Intelligent Transportation System,” vol. 2, no. 2, pp. 933-946, 2018.

[6] A. M. Luthfi, N. Karna, and R. Mayasari, "Google Maps API Implementation On IOT Platform For Tracking an Object Using GPS," in 2019 IEEE Asia Pacific Conference on Wireless and Mobile (APWiMob), Nov. 2019, pp. 126-131, doi: 10.1109/APWiMob48441.2019.8964139.

[7] A. Mounika, "IOT Based Vehicle Tracking and Monitoring System Using GPS and GSM," International Journal of Recent Technology and Engineering (IJRTE), vol. 8, no. 2S11, pp. 2399-2403, Nov. 2019, doi: 10.35940/ijrte.B1275.0982S1119.

[8] T. Srivastava, "Real-time tracking of water tanker using GPS, GSM , and Arduino," International Journal of Advance Research, Ideas and Innovations in Technology, vol. 6, no. 3, pp. 340-344, 2020.

[9] E. Aytaç Adali, A. Tuş Işik, And N. Kundakçi, "Tourist Hotel Location Ion With Analytic Hierarchy Process," Journal of Life Economics, vol. 2, no. 3, pp. 47-58, 2015, doi: 10.15637/jlecon.82.

[10] R. H. Kouchaksaraei, S. H. Zolfani, and M. Golabchi, "Glasshouse locating based on SWARA-COPRAS approach," International Journal of Strategic Property Management, vol. 19, no. 2, pp. 111-122, 2015, doi: 10.3846/1648715X.2015.1004565.

[11] M. A. Nazari, A. Aslani, and R. Ghasempour, "Analysis of solar farm site selection based on TOPSIS approach," International Journal of Social Ecology and Sustainable Development (IJSESD), vol. 9, no. 1, pp. 12-25, 2018, doi: 10.4018/IJSESD.2018010102.

[12] S. H. Zolfani, S. Mosharafiandehkordi, and V. Kutut, "A pre-planning for hotel locating according to the sustainability perspective based on bwm-waspas approach," International Journal of Strategic Property Management, vol. 23, no. 6, pp. 405419, 2019, doi: 10.3846/ijspm.2019.10844

[13] T. Şahin, S. Ocak, and M. Top, "Analytic hierarchy process for hospital site selection," Health Policy and Technology, vol. 8, no. 1, pp. 42-50, 2019, doi: 10.1016/j.hlpt.2019.02.005

[14] S. H. Zolfani, M. Yazdani, A. E. Torkayesh, and A. Derakhti, "Application of a gray-based decision support framework for location selection of a temporary hospital during COVID-19 pandemic," Symmetry (Basel)., vol. 12, no. 6, 2020, doi: 10.3390/SYM12060886.

[15] E. A. Adalı and A. Tuş, "Hospital site selection with distance-based multi-criteria decision-making methods," International Journal of Healthcare Management, vol. 14, no. 2, pp. 534-544, 2021, doi: 10.1080/20479700.2019.1674005.

[16] R. S. Rosli, M. H. Habaebi, and R. Islam, "On the analysis of received signal strength indicator from ESP8266," vol. 8, no. 3, 2019, doi: 10.11591/eei.v8i3.1511.

[17] A. Murtaza, A. Jalil, R. Mohamad, N. M. Anas, and M. Kassim, "Implementation of vehicle ventilation system using NodeMCU ESP8266 for remote monitoring," Bulletin of Electrical Engineering and Informatics, vol. 10, no. 1, pp. 327-336, 2021, doi: 10.11591/eei.v10i1.2669.

[18] W. S. Pambudi, E. Alfianto, A. Rachman, and D. P. Hapsari, "Simulation design of trajectory planning robot manipulator," Bulletin of Electrical Engineering and Informatics, vol. 8, no. 1, pp. 196-205, 2019, doi: 10.11591/eei.v8i1.1179.

[19] A. A. Najib, R. Munadi, N. Bogi, and A. Karna, "Security system with RFID control using E-KTP and internet of things," Bulletin of Electrical Engineering and Informatics, vol. 10, no. 3, pp. 1436-1445, 2021, doi: 10.11591/eei.v10i3.2834.

[20] K. R. Trivedi and D. P. Mistry, "Integration of GPS and GSM for the Weather Monitoring System,” vol. 1, no. 3, pp. 2-5, 2012.

[21] A. K. Mahamad, S. Saon, H. Hashim, and M. A. Ahmadon, "Cloud-based people counter," Bulletin of Electrical Engineering and Informatics, vol. 9, no. 1, pp. 284-291, 2020, doi: 10.11591/eei.v9i1.1849.

[22] S. I. Abdullahi, M. H. Habaebi, and N. A. Malik, "Intelligent flood disaster warning on the fly: developing IoT-based management platform and using 2-class neural network to predict flood status," Bulletin of Electrical Engineering and Informatics, vol. 8, no. 2, pp. 706-717, 2019, doi: 10.11591/eei.v8i2.1504

[23] S. Sherif, W. H. Tan, C. P. Ooi, A. Sherif, and S. Mansor, "LoRa driven medical adherence system," Bulletin of Electrical Engineering and Informatics, vol. 9, no. 6, pp. 2294-2301, 2020, doi: 10.11591/eei.v9i6.2195.

[24] J. H. Majeed and Q. Aish, "A remote patient monitoring based on WBAN implementation with internet of thing and cloud server," Bulletin of Electrical Engineering and Informatic,s vol. 10, no. 3, pp. 1640-1647, 2021, doi: 10.11591/eei.v10i3.1813.

[25] M. Keshavarz-Ghorabaee, M. Amiri, E. K. Zavadskas, Z. Turskis, and J. Antucheviciene, "Determination of objective weights using a new method based on the removal effects of criteria (Merec)," Symmetry (Basel)., vol. 13, no. 4, pp. 1-20, 2021, doi: 
10.3390/sym13040525.

[26] C. R. Pattnaik, S. N. Mohanty, S. Mohanty, J. M. Chatterjee, B. Jana, and V. García-Díaz, "A fuzzy multi-criteria decisionmaking method for purchasing life insurance in india," Bulletin of Electrical Engineering and Informatics, vol. 10, no. 1, pp. 344356, 2021, doi: 10.11591/eei.v10i1.2275.

[27] V. B. S. Prasath et al., "Distance and Similarity Measures Effect on the Performance of K-Nearest Neighbor Classifier -- A Review," Big Data, pp. 1-39, 2017, doi: 10.1089/big.2018.0175.

[28] V. Kumar, J. K. Chhabra, and D. Kumar, "Performance Evaluation of Distance Metrics in the Clustering Algorithms," INFOCOMP Journal of Computer Science, vol. 13, no. 1, pp. 38-51, 2014.

[29] R. Suwanda, Z. Syahputra, and E. M. Zamzami, "Analysis of Euclidean Distance and Manhattan Distance in the K-Means Algorithm for Variations Number of Centroid K," Journal of Physics: Conference Series, Volume 1566, 4th International Conference on Computing and Applied Informatics 2019 (ICCAI 2019), Medan, Indonesia, 26-27 November 2019, vol. 1566, no. 1, 2020, doi: 10.1088/1742-6596/1566/1/012058.

[30] R. A. Hamid and M. S. Croock, "A developed GPS trajectories data management system for predicting tourists' POI," TELKOMNIKA (Telecommunication, Computing, Electronics and Control), vol. 18, no. 1, pp. 124-132, 2020, doi: 10.12928/TELKOMNIKA.V18I1.13006.

[31] O. R. Kuzichkin, G. S. Vasilyev, A. V. Grecheneva, E. V. Mikhaleva, M. D. Baknin, and D. I. Surzhik, "Application of phasemetric compensation method for geoelectric control of near-surface geodynamic processes," Bulletin of Electrical Engineering and Informaticss, vol. 9, no. 3, pp. 898-905, 2020, doi: 10.11591/eei.v9i3.1727.

[32] P. Megantoro, B. A. Pramudita, P. Vigneshwaran, and A. Yurianta, "Real-time monitoring system for weather and air pollutant measurement with HTML-based UI application," Bulletin of Electrical Engineering and Informatics, vol. 10, no. 3, pp. 16691677, 2021, doi: 10.11591/eei.v10i3.3030.

[33] A. S. Cabezuelo, "Generation of infectious disease alerts through the use of geolocation," Bulletin of Electrical Engineering and Informatics, vol. 9, no. 4, pp. 1533-1541, 2020, doi: 10.11591/eei.v9i4.1945.

[34] A. Moutaouakkil and S. Mbarki, "Php modernization approach generating kdm models from php legacy code," Bulletin of Electrical Engineering and Informatics, vol. 9, no. 1, pp. 247-255, 2020, doi: 10.11591/eei.v9i1.1269.

[35] S. A. Zulkiflie, N. Kamaruddin, and A. Wahab, "Dynamic navigation indoor map using wi-fi fingerprinting mobile technology," Bulletin of Electrical Engineering and Informatics, vol. 9, no. 2, pp. 739-746, 2020, doi: 10.11591/eei.v9i2.2066.

[36] P. Mookdarsanit and L. Mookdarsanit, "The COVID-19 fake news detection in Thai social texts," Bulletin of Electrical Engineering and Informatics, vol. 10, no. 2, pp. 988-998, 2021, doi: 10.11591/eei.v10i2.2745.

[37] T. Wahyuningrum, C. Kartiko, A. C. Wardhana, and T. G. Soares, "Revised web impact factor analysis of Timor Leste University website during COVID-19 pandemic," Bulletin of Electrical Engineering and Informatics, vol. 10, no. 3, pp. 1678-1686, 2021, doi: 10.11591/eei.v10i3.3034.

\section{BIOGRAPHIES OF AUTHORS}
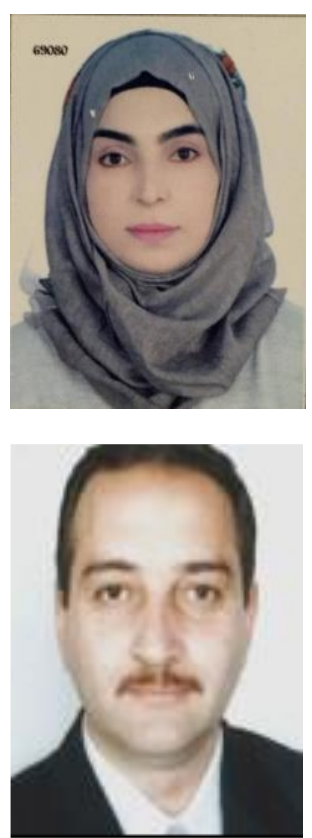

Abeer Hadi (D) 8 SC P received his Bachelor degree in Computer Engineering, from Al Mustansiriyah University, Iraq in 2011. His interests involve IoT technology and designing websites. She can be contacted at email: egma019@uomustansiriyah.edu.iq.

Mahmood Zaki Abdullah (D) SC $\mathrm{P}$ is one of an associate professor doctor at Mustansiriya University Baghdad-Iraq. He holds a $\mathrm{PhD}$ degree in computer engineering and has many books in computer engineering, networks, smart systems and information technology.He can be contacted at email: drmzaali@uomustansiriyah.edu.iq. 